\title{
A Full Coverage Reconstruction Algorithm for the Electromagnetic Map Based on Affinity Propagation Clustering
}

Dongming Lin ( $\sim 2747240743 @ q q . c o m$ )

National University of Defense Technology https://orcid.org/0000-0002-0387-7663

Hongjun Wang

National University of Defense Technology

\section{Research}

Keywords: Electromagnetic Maps, Reconstruction, Affinity Propagation Clustering, Kriging, Domain mapping

Posted Date: November 30th, 2021

DOI: https://doi.org/10.21203/rs.3.rs-1098471/v1

License: (c) (i) This work is licensed under a Creative Commons Attribution 4.0 International License.

Read Full License 
Correspondence:

lindongming@nudt.edu.cn College of Engineering, National University of Defense Technology, Hefei, China

Full list of author information is available at the end of the article

\begin{abstract}
Considering the reconstruction of electromagnetic maps without the prior information of electromagnetic propagation environment in the target area, a new algorithm based on affinity propagation clustering is proposed to complete the electromagnetic map reconstruction of the target area from points to surfaces and then from points and surfaces to a larger surface. Firstly, according to the actual situation, the target area is reasonably divided into grids. Electromagnetic data is sampled by distributed sensing nodes, and a certain number of sample points are selected for affinity propagation clustering to determine the locations of centers of sample points. Secondly, for the incomplete sample data, the Kriging algorithm is used to reconstruct the small circular electromagnetic maps. The class center is the center of the circle and the radius is certain. After that, the obtained small area electromagnetic map data and the data obtained from the sample points are used for domain mapping processing, and the electromagnetic data of a larger area of the target area is obtained. Finally, the overall electromagnetic map is reconstructed through data fusion. The simulation results show that the proposed algorithm is better than several interpolation algorithms. When sample points account for 0.1 of total data points, the RMSE of the result is less than 1.5.
\end{abstract}

Keywords: Electromagnetic Maps; Reconstruction; Affinity Propagation Clustering; Kriging; Domain mapping

\section{Introduction}

The electromagnetic map is a kind of graphics or images that can reflect electromagnetic information in space, time and frequency domain when the signal is in the complex geographical environment. It is a promising technology in cognitive radio and frequency use [1] [2]. Its connotation covers radio, radar, satellite and other electromagnetic signal radiation sources.

The research into electromagnetic maps is mainly about radio environment maps [3]. Before the reconstruction of radio environment map, it is necessary to obtain the radio information of each geographical location. The traditional method is based on measurement by manual work, but it requires a lot of manpower and material resources. Moreover, it is not achievable in inaccessible regions. With the development of distributed wireless network technology, the use of small unmanned platform equipped with sensing equipment to detect the radio signal in the target region has become the preferred way to obtain data [4]. However, among the data obtained by the sensing nodes, only the received signal power value at the position of the 
sensing node itself is accurate. The assumption that sensing nodes can accurately obtain the data within the sensing range is only applicable to application scenarios with sufficient prior information. However, in practical applications, it is difficult to obtain the prior information. Therefore, it is necessary to study how to reconstruct the radio environment maps in the absence of prior information.

In recent years, geological interpolation algorithms such as Inverse Distance Weighting algorithm(IDW) [5] and Kriging algorithm(KGA) [6] have been used in the reconstruction of radio environment maps. In literature [5], the classical IDW and the Modified Shepard's Method(MSM) are used to reconstruct the radio environment map, and the radio interference field is measured by using the reconstructed radio environment map. The proposed algorithm can make use of the sampling data effectively and has strong robustness. However, when the sensing nodes are close to the radiation source, the error of the interpolation will increase significantly. Moreover, the IDW itself has some shortcomings such as lack of data smoothness and being easily influenced by outliers. Literature [6] focuses on the reconstruction of radio environment maps. Since KGA is applied, the radio environment maps reconstructed are featured with improved smoothness and higher accuracy. In addition, the Nearest Neighbor algorithm(NN) is applied to the reconstruction of the radio environment maps. Generally speaking, KGA has the characteristics of high interpolation accuracy and good data smoothness, but it also has its own drawbacks, such as sensitivity to outliers. A small amount of false information will cause the sharp downfall in accuracy, too [7]. NN is better for filling regions with no values, but there is a problem of discontinuous numerical changes. In addition, the Local Polynomial algorithm(LP) [8] and the Minimum Curvature algorithm(MC) [9] are also commonly used interpolation algorithms, which can also be applied to the reconstruction of radio environment maps. Reconstructing a radio environment map by interpolation algorithms is also called the direct method. Of course, there are indirect methods and hybrid methods, too. The indirect method reconstructs the radio environment maps by the radio wave propagation model [10]. Literature [10] discusses the role of the propagation model and the accuracy of the reconstructed radio environment map. But this kind of algorithm often requires channel characteristics of the target region. In addition to the transmission loss in the free space, the algorithm also needs to consider the shadow fading, the diffraction loss, as well as the selection of appropriate experience model. The determination of parameters and model selection are both highly demanding. Reconstructing radio environment maps based on transmitter locations also falls into this category [11]. The hybrid method is a method that combines the propagation model(or transmitter locations) and the interpolation algorithm to reconstruct radio environment maps [12, 13]. Due to the fact that it combines both direct and indirect methods, the accuracy is high, but the complexity also increases.

Projection Onto Convex Sets (POCS), originally used in the super-resolution reconstruction of images, has been introduced to the reconstruction of seismic data. In literature [14], POCS is used in the reconstruction of vacant data through Fourier transform. This method can directly complete the reconstruction of data iteratively, and it has low complexity and stable effect in the reconstruction of regular missing data. Simulation results show that the effect is better than that of KGA, IDW and 
$\mathrm{MC}$, but it is not applicable to the reconstruction of irregular missing data. In the reconstruction of seismic data through POCS, the basic idea is to map the existing domain of the data, process the data in another domain, and reconstruct the defect data through iterations [15].

\section{Method}

Among the existing electromagnetic map construction methods, the Kriging algorithm has high complexity and the accuracy of the data obtained is greatly affected by the outliers, and the Inverse Distance Weighting algorithm is greatly affected by the outliers, and the accuracy of the obtained results is low. When reconstruct large scale data, the accuracy of the result is not high. The application scenarios of other algorithms such as Nearest Neighbor algorithm and Local Polynomial algorithm are quite limited. Moreover, the signal propagation environment in the area is often unknowable, so the data obtained within the sensing range of the sensing node cannot be regarded as accurate data. In order to achieve high-precision reconstruction of the electromagnetic map in the absence of prior information, and to minimize the number of sensing nodes required, considering the high accuracy of KGA [6] and referring to the advantages of mapping gravity and magnetic data to another domain [14] [15], this paper proposes a new algorithm based on affinity propagation clustering including three parts. The first part is affinity propagation clustering algorithm(APCA), the second one is Kriging algorithm(KGA), and the last one is domain mapping algorithm(DPA). Due to the wide variety of electromagnetic maps and lack of space, we just study the reconstruction of the electromagnetic signal receiving intensity map (hereinafter referred to as the electromagnetic map) and take Reference Signal Receiving Power (RSRP) as the research object.

\section{Implementation of the algorithm}

The main framework of the algorithm is: according to the actual situation, the target area is divided into grids in advance, the geographical position of each grid is obtained and the total amount of electromagnetic data is determined. Reasonably determining the amount of data can effectively avoid data redundancy and facilitate experiment. Then a certain number of sensing nodes are deploy in the target area to obtain the electromagnetic data at the corresponding location, that is, sample points. After that a certain number of sample points are selected to perform affinity propagation clustering on their geographic locations to obtain the cluster center, and Kriging algorithm is used to reconstruct small-area electromagnetic maps with the location of the cluster center as the center and a certain radius. Then, the electromagnetic data is subjected to two-dimensional Fourier transform, and the data is mapped from the time-space domain to the frequency-wave number domain for processing. Finally, the data is inversely mapped back to the original existence domain. The final overall electromagnetic map data of the target area is obtained by fusing the original data, the data obtained by the Kriging algorithm, and the data obtained by the domain mapping processing.

\subsection{Affinity propagation clustering algorithm}

It is a convenient and effective method to obtain radio data in the target region by using distributed sensing nodes [16]. A certain number of sensing nodes are 
randomly scattered or deployed in a specific way in the target region, and the electromagnetic environment is sampled by the sensing nodes. Then a certain number of sampling points are selected as cluster points, and the proposed algorithm processes them with APCA to obtain the geographic location of the corresponding cluster centers.

APCA is a kind of clustering algorithm based on message passing [17]. The algorithm regards the data set as a node network, passes two kinds of messages named responsibility $r(i, k)$ and availability $a(i, k)$ among each node, and adjusts the cluster centers continuously by using the messages to maximize the similarity of the node network. Then corresponding cluster centers are obtained. Since the algorithm does not need to specify the number of cluster classes in advance and the cluster centers are true points, which is applicable to the application scenarios with insufficient prior information, the proposed algorithm uses it for clustering processing.

The algorithm realizes the update of messages through the following formulas.

$$
\begin{aligned}
& r(i, k)=s(i, k)-\max _{k^{\prime} \neq k}\left[a\left(i, k^{\prime}\right)+s\left(i, k^{\prime}\right)\right] \\
& a(i, k)=\left\{\begin{array}{cc}
\min \left\{0, r(k, k)+\sum_{i^{\prime} \notin(i, k)}\left[0, r\left(i^{\prime}, k\right)\right]\right\} & i \neq k \\
\sum_{i^{\prime} \neq k} \max \left[0, r\left(i^{\prime}, k\right)\right] \quad i=k &
\end{array}\right.
\end{aligned}
$$

Where $r(i, k)$ stands for the responsibility when the $k$ th sample becomes the center of the $i$ th sample, and $a(i, k)$ stands for the availability when the $i$ th sample chooses the $k$ th sample as the center. $s(i, k)$ stands for the similarity between the $k$ th sample and the $i$ th sample, and the algorithm chooses the negative squared Euclidean distance between the $i$ th sample and the $k$ th sample as its value. $s(i, i)$ stands for the preference when the $i$ th sample is chosen as the center, whose value is the median of the similarity. The two kinds of messages are updated as follows.

$$
\left\{\begin{array}{l}
r_{\text {new }}(i, k)=\lambda r_{\text {old }}(i, k)+(1-\lambda) r_{\text {new }}(i, k) \\
a_{\text {new }}(i, k)=\lambda a_{\text {old }}(i, k)+(1-\lambda) a_{\text {new }}(i, k)
\end{array}\right.
$$

$\lambda$ is the damping factor used to guarantee the algorithm convergence, and its value is 0.9 .

\subsection{Kriging algorithm}

KGA can get the more precise output of RSRP as follows [18].

$$
y_{\text {out }}(h)=\mathbf{w}^{\mathrm{T}}(h) \boldsymbol{\alpha}+e(h)
$$

Where $y_{\text {out }}(h)$ is the value of RSRP, and $h$ is the location. $\mathbf{w}(h)=\left[w_{1}(h), w_{2}(h), \ldots, w_{N}(h)\right]^{\mathrm{T}}$ is the $N$ selected functions that make up the regression model. $\boldsymbol{\alpha}=\left[\alpha_{1}, \alpha_{2}, \ldots, \alpha_{N}\right]^{\mathrm{T}}$ is the parameters of the model. $e(h)$ is a stochastic process satisfying the following conditions.

$$
\left\{\begin{array}{c}
E[e(h)]=0 \\
E\left[e\left(h_{i}\right) e\left(h_{j}\right)\right]=\tau^{2} R\left(\theta, h_{i}, h_{j}\right)
\end{array}\right.
$$


Where $h_{i}, h_{j}$ are both input data, $E[]$ is calculating the mean value of the variate, and $R\left(\theta, h_{i}, h_{j}\right)$ is the correlation model, $\tau^{2}$ is the variance of $e(h)$, and $\theta$ is the parameter of the correlation model. Suppose a set of sampling points $\left[s_{1}, \ldots, s_{q}\right]$, its values are $\mathbf{Y}=\left[y_{1}, \ldots, y_{q}\right]^{\mathrm{T}}$. So an equation is got.

$$
\left\{\begin{array}{c}
\hat{\boldsymbol{\alpha}}=\left(\mathbf{F}^{\mathrm{T}} \mathbf{R}^{-1} \mathbf{F}\right)^{-1} \mathbf{F}^{\mathrm{T}} \mathbf{R}^{-1} \mathbf{Y} \\
\hat{\tau^{2}}=\frac{1}{q}(\mathbf{Y}-\mathbf{F} \hat{\boldsymbol{\alpha}})^{\mathrm{T}} \mathbf{R}^{-1}(\mathbf{Y}-\mathbf{F} \hat{\boldsymbol{\alpha}})
\end{array}\right.
$$

In the above formula, $\hat{\boldsymbol{\alpha}}$ is the estimated value of $\boldsymbol{\alpha}, \hat{\tau^{2}}$ is the estimated value of $\tau^{2}$. F is a $q \times N$ matrix containing $w\left(s_{i}\right)$, for $i \in[1, q]$. $\mathbf{R}$ is $q \times q$ correlation matrix of the sampling points containing $R_{i j}=R\left(\theta, s_{i}, s_{j}\right)$. The correlation function is defined as:

$$
R(\theta, \mu, h)=\prod_{j=1}^{n} R_{j}\left(\theta, \mu_{j}-h_{j}\right)
$$

Where $n$ is the dimension of the sampling points. And the algorithm needs to guarantee that the following is true.

$$
\min _{\theta}\left[\xi(\theta)=|\mathbf{R}|^{\frac{1}{q}} \tau^{2}\right]
$$

$|\mathbf{R}|$ is calculating the determinant of the matrix. Here, the proposed algorithm adapts the spherical model.

$$
R_{j}\left(\theta, d_{j}\right)=1-1.5 \varphi_{j}+0.5 \varphi_{j}^{3} \quad \varphi_{j}=\min \left\{1, \theta_{j}\left|d_{j}\right|\right\}
$$

$d_{j}=\mu_{j}-h_{j}$. Finally, the predicted value of RSRP in the unknown location is shown as follows.

$$
\left\{\begin{array}{c}
\hat{y o u}(h)=\mathbf{w}^{\mathrm{T}}(h) \hat{\boldsymbol{\alpha}}+\mathbf{r}^{\mathrm{T}}(h) \mathbf{R}^{-1}(\mathbf{Y}-\mathbf{F} \hat{\boldsymbol{\alpha}}) \\
\mathbf{r}(h)=\left[R\left(\theta, h, s_{1}\right), \ldots, R\left(\theta, h, s_{q}\right)\right]^{\mathrm{T}}
\end{array}\right.
$$

After that, the RSRP values of unknown locations in some circular regions whose centers are cluster centers and radiuses are certain are predicted, so that the smallarea map data can be obtained. If the number of sensing nodes is small, the effect in reconstruction can be optimized by increasing the reconstructing radius and the proportion of sample points for clustering to the total sampling points.

\subsection{Domain mapping algorithm}

The predicted values of RSRP obtained from KGA are filled into the corresponding positions of the incomplete data sampled by the sensing nodes, and then it is used as the input data of the domain mapping processing.

First, the data is extended, then the data is filled with zeros, and the extraction matrix is obtained.

$$
S(x, y)= \begin{cases}1 & d_{0}(x, y)=N a N \\ 0 & d_{0}(x, y) \neq N a N\end{cases}
$$


$d_{0}(x, y)$ is the data whose position is $(x, y)$ in the incomplete data to be processed, $N a N$ shows that data is missed in that position.

Second, the proposed algorithm processes the data by eq(12).

$$
d_{t}(x, y)=F^{-1}\left\{T_{k}\left[F\left(d_{0}(x, y)+S(x, y) d_{t-1}(x, y)\right)\right]\right\}
$$

Where $t \in[1, N]$ is the number of the iterations. $F$ is two-dimensional Fourier transform, and $F^{-1}$ is the inverse Fourier transform, $T_{k}$ is the circular low-pass filter for $k \in[1, \ldots, \kappa]$, and $\kappa$ is the cut-off wave number, $N$ is the total number of iterations.

$$
T_{k}(\alpha, \beta)= \begin{cases}1 & \alpha^{2}+\beta^{2} \leq \delta^{2} \\ 0 & \alpha^{2}+\beta^{2}>\delta^{2}\end{cases}
$$

$\alpha$ is the abscissa and $\beta$ is the ordinate relative to the data center, $\delta$ is the radius of the filter. The data obtained in this step is $d_{s}(x, y)$.

Third, process $d_{s}(x, y)$, and the final results are got.

$$
\left\{\begin{array}{c}
d_{\text {all }}(x, y)=d_{0}(x, y)+S(x, y) d_{s}(x, y) \\
x \in\left[\text { broad, broad }+x_{\text {ori }}\right] \\
y \in\left[\text { broad, broad }+y_{\text {ori }}\right]
\end{array}\right.
$$

broad is the length of extension. $x_{\text {ori }}$ is the amount of data in a single row, $y_{\text {ori }}$ is the amount of data in a single column, $d_{a l l}(x, y)$ is the complete data we need.

The procedure of DPA is shown as follows. The step of calculating radial average

Figure 1 Data processing procedure of domain mapping

power spectrum includes three parts.

1. Two-dimensional Fourier transform is used to map the data to the wave number domain and the power spectrum is calculated.

2. We set the fundamental frequency, and we take the data center as the center of a circle. We also take integer multiples of the fundamental frequency as the radiuses to construct several concentric rings, and the average value of power spectrum within the range of each ring is calculated.

3. Integer multiples of the fundamental frequency are taken as the abscissas and the average value of power spectrum in each ring that are taken the log of are taken as the ordinates to get the radial average power spectrum.

Here, we propose a new method to determine the cut-off wave number. We think it is the first wave number when the radial average power spectrum is less than or equal to one fifth of the maximum value.

We can obtain the complete electromagnetic map data by filling the values obtained by DPA into the corresponding positions of the input data of DPA. 


\section{Simulations and analyses}

\subsection{Simulations}

At present, many scholars who study radio environment maps regard the Received Signal Strength Indicator (RSSI) as the object of reconstruction. This indicator represents the strength of the signal received by each terminal in the wireless communication network. RSRP represents the average power of the reference signal carried by a symbol in the terminal of a wireless communication network. Considering that RSRP measuring the electromagnetic signal strength is more accurate than RSSI, this paper regards RSRP as the relevant parameter to describe the regional signal strength distribution.

Without loss of generality, the simulation takes the LTE mobile communication network as the target network and extracts the radio coverage in a $4,000 \mathrm{~m} \times 4,000 \mathrm{~m}$ region of the Brussels map as the experimental data. The original electromagnetic map is shown in Fig.2. There are three base stations (hereinafter referred to as radiation sources) in this region, with a maximum transmitting power of $43 \mathrm{dBm}$, a frequency band width of $2110 \mathrm{FDD}-10 \mathrm{MHz}$, and a cell radius of $350 \mathrm{~m}$. Since Atoll is a professional software for electromagnetic propagation simulation, its results are very accurate, so this article uses it for data generation. The model used is the OkumuraHata model. Because the area is an actual geographic area with mountain uplifts and rivers and other features, it can reflect the true electromagnetic propagation to a greater extent. After rasterization of the target region, 40,000 grids can be obtained, and 4,000 low-cost sensing nodes are randomly scattered in the region. That is, sampling points (sensing nodes) account for 0.1. The experimental platform is Core i7, and the relevant software and versions used are Matlab R2018b, Atoll 2.8.0 and Surfer 14.

Figure 2 The original electromagnetic map

Fig.3. is the results of clustering with 400 clustering points, and different colors stands for different classes.

Figure 3 Results of clustering

After obtaining the cluster centers, the proposed algorithm uses cluster centers as the circular centers and a radius of 200 meters to reconstruct small-area electromagnetic maps to complete the data acquisition from points to surfaces. The results are circular regions as shown in Fig.4.

Figure 4 The reconstruction of small-area electromagnetic maps.

The data obtained from KGA is filled to the corresponding vacant positions of the original sampling data, and then the data is mapped from the time-space domain to the frequency-wave number domain for processing. The length of the extended edge is 20 pixel units. Then the result of DPA is obtained by inverse mapping. That is, data acquisition from points and small surfaces to the larger surface is completed. Fig. 5 is the result. 
Figure 5 The result of DPA.

\subsection{Analyses}

The root mean square error (RMSE) can effectively measure the average deviation between the estimated value and the original one, and it can also quantitatively describe the accuracy of the estimated data obtained. The determination coefficient $R^{2}$ can effectively reflect the degree of closeness between the result of reconstruction and the actual one. When the determination coefficient is bigger, the data distribution of the reconstruction result is closer to that of real data. That is, the consistency between the radio coverage of the obtained map and that of the real situation is higher. Moreover, when the available nodes are less than expected, the reconstruction effect of the algorithm should not change too much. In other words, the algorithm should have good robustness. It can be judged by the RMSE of the algorithm and its amplitude of variation when reducing the proportion of sampling points. Therefore, when studying the effect of the proposed algorithmm, the paper evaluates the effect from RMSE, determination coefficient and the robustness.

$$
R M S E=\sqrt[2]{\frac{\sum_{i=1}^{l}(\hat{D}-D)^{2}}{l}}
$$

$l$ is the total number of electromagntic data, $\hat{D}$ is the estimated value, $D$ is real one.

$$
\left\{\begin{array}{c}
\bar{z}=\frac{1}{l} \sum_{i=1}^{l} z_{i} \\
S_{t o t}=\sum_{i=1}^{l}\left(z_{i}-\bar{z}\right)^{2} \\
S_{r e s}=\sum_{i=1}^{l}\left(\hat{z}_{i}-\bar{z}\right)^{2} \\
R^{2}=1-\frac{S_{r e s}}{S_{\text {tot }}}
\end{array}\right.
$$

$z_{i}$ is the $i$ th data, $\bar{z}$ is the average value of $z, S_{\text {tot }}$ is the total sum of squares, $S_{\text {res }}$ residual sum of squares, $R^{2}$ is the determination coefficient.

In order to research into the effect of the proposed algorithm, KGA, IDW, MSM, NN and LP are applied to the reconstruction of electromagnetic maps and compared with the proposed algorithm. Simulation results are shown in Fig. 6. Among them, Fig. 6 (a) is the result of KGA, Fig. 6(b) is the result of IDW, Fig. 6 (c) is the result of MSM, Fig. 6 (d) is the result of NN, Fig. 6 (e) is the result of LP, and Fig. 6 (f) is the result of the proposed algorithm. A complete electromagnetic map is reconstructed by combining the sampling data and the results of KGA and DPA.

Figure 6 Comparative results of reconstruction

The result obtained by KGA has a poor effect in the region where radiation resources are located. The form of the isomagnetic line is very messy, and there are many abnormal values. It is impossible to clearly show the received power 
distribution in that region, so the map quality is barely satisfactory. According to the result obtained by IDW, the isomagnetic line of the map is very messy everywhere. There are many abnormal values and serious bull's-eye effect, so the map quality is worse. As to the result obtained by MSM, it is clear and the form of the isomagnetic line is better. However, the bull's-eye effect and some abnormal values still exist. On the whole, the map quality is better. The map obtained by NN is messy, and the isomagnetic line undulates. The numerical change is discontinuous. The quality of the map is worse. Although the form of the isomagnetic line in the result obtained by LP is clear with no bull's-eye effect, the abnormal values exist in patches, and the electromagnetic distribution of the region where radiation sources are located cannot be accurately displayed. The quality of the map is worse. In the result of the proposed algorithm, the form of the isomagnetic line is satisfactory especially in the region where the radiation sources are located, so the change of the received power in this region is better described. Additionally, there are fewer abnormal values and no bull's-eye effect. The quality of the reconstructed map is ideal. Apart from the quality of the map, the accuracy is also the key to the measurement of the effectiveness of the algorithm. TABLE 1 shows the RMSEs and the determination coefficients of six algorithms in the case of 0.1 sampling data.

Table 1 Analyses Of Performance 1

\begin{tabular}{ccccccc}
\hline & this paper & IDW & KGA & NN & MSM & LP \\
\hline RMSE $/ \mathrm{dBm}$ & 0.8622 & 2.2166 & 0.9386 & 1.0852 & 1.0887 & 1.5984 \\
$R^{2}$ & 0.9823 & 0.8832 & 0.9791 & 0.9720 & 0.9718 & 0.9393 \\
\hline
\end{tabular}

As can be seen from TABLE 1, the proposed algorithm has the smallest RMSE and the largest determination coefficient, so the average deviation degree between the result obtained by the algorithm and the original one is the smallest, and the data distribution of the reconstruction result is the closest to that of the original data, making it the algorithm with the highest accuracy among the six. Fig.7 and Fig.8 shows the RMSE of the results obtained by each algorithm when the proportion of sampling points decreases from 0.5 to 0.1 and the proportion of sampling points decreases from 0.05 to 0.01 .

Figure 7 Analyses of robustness 1

Figure 8 Analyses of robustness 2

In Fig7, as the proportion of sampling points decreases, the amplitude in the RMSE of the proposed algorithm is increased by 0.3274 . Moreover, except the case that the proportion of sampling points is 0.30, the RMSEs are all the smallest. Even though when the sampling points account for 0.3, the RMSE of the proposed algorithm is almost the same as the smallest one. From Fig.8, it is easily seen that the RMSE of the proposed algorithm is 0.4851 , the second smallest. But it is not much different from the minimum value of 0.4244 , and the RMSE of the proposed algorithm is smaller than that of the other algorithms'. Therefore, the proposed algorithm has better robustness and stable effect. 
When the number of sampling points is small, the algorithm considers expanding the reconstruction radius and increasing the proportion of clustering samples to sampling points to optimize the effect. After verification of a large number of experimental results, it can be found that when the number of sampling nodes accounts for 0.05 or more of the total electromagnetic data, the reconstruction radius that is about 10 times the length of the divided grid can have a good effect, when the sampling nodes account for less than 0.05 of the total amount of electromagnetic data, the reconstruction radius is about 25 times the grid length, it will have a good effect. If the radius is too large or too small, the quality of the map will deteriorate and the accuracy of the data will decrease. The number of cluster samples generally does not exceed 0.025 of the total electromagnetic data.

Now, we discuss the effect of proposed algorithm when the proportion of the sampling data is 0.01 .

Table 2 Analyses Of Performance 2

\begin{tabular}{ccccccc}
\hline & this paper & IDW & KGA & NN & MSM & LP \\
\hline RMSE $/ \mathrm{dBm}$ & 1.4770 & 3.1657 & 1.4814 & 2.1890 & 2.3159 & 3.4493 \\
$R^{2}$ & 0.9482 & 0.7619 & 0.9479 & 0.8861 & 0.8725 & 0.7173 \\
\hline
\end{tabular}

From the results, it is clearly indicated that the proposed algorithm has the smallest RMSE(1.4770) and the largest determination coefficient(0.9482), so the average deviation between the results obtained by it and the original value is the smallest, and the data distribution of the obtained result is closer to the real situation.

In summary, the effect of our algorithm is better than the other five algorithms.

\section{Results and discussion}

This paper proposes a new full coverage reconstruction algorithm based on affinity propagation clustering to reconstruct the electromagnetic map. When information of the signal propagation environment in the target area is unknown, sampling data is only relying on the distributed node network and we can not calculate other unknown data by the propagation equation. At this situation, our algorithm can be used to effectively reconstruct the electromagnetic map to know the other data. The results is not only highly precise with the lowest RMSE value and the clearest image, but also has strong robustness. Moreover, it requires a small number of sensing nodes and low complexity. Even though when the sample data points account for 0.1 of total data points, it can make the RMSE value less than 1.5. The simulation results show that the effect of our algorithm is better than the existing Kriging algorithm, Inverse Distance Weighting algorithm and other algorithms.

However, our algorithm needs accurate data. That is to say, if data obtained by sensors is not correct, the effect can not be guaranteed. And when the sample data points account for 0.1 of total data points, the accuracy of results can be improved, and the reconstruction quality need to be improved, too. Therefore, these are the research of next stage.

Nowadays, the spectrum resource is very sparse with low using rate, but the electromagnetic map can be used for spectrum management and utilization. So what we research has vital importance to the world. 


\section{Appendix}

Abbreviations

APCA:Affinity Propagation Clustering Algorithm; KGA:Kriging Algorithm; DPA:Domain Mapping Algorithm; RSRP:Reference Signal Receiving Power; RSSI:Received Signal Strength Indicator; RMSE:Root Mean Square Error; IDW:Inverse Distance Weighting; MSM:Modified Shepard's Method; NN:Nearest Neighbor; LP:Local Polynomial.

Authors' contributions

Dongming Lin: Writing and editing;Data analysis. Hongjun Wang: editing. The authors read and approved the fnal manuscript.

Authors' information

Dongming Lin(1997). Male, Fujian Province, China. Research direction: Distributed sensor

networks.lindongming@nudt.edu.cn.

Hongjun Wang(1968). Male, Jiangsu Province, China. Research direction: Distributed sensor networks; Cognitive communication.hongjun-wang@163.com.

Funding

The authors received no fnancial support for the research, authorship, and/or publication of this article.

Availability of data and materials

Data sharing does not apply to this article because no data set was generated or analyzed during the current research period.

Declarations

Ethics approval and consent to participate

This article is ethical, and this research has been agreed.

Consent for publication

The picture materials quoted in this article have no copyright requirements, and the source has been indicated.

Competing interests

The authors declare that they have no competing interests.

Acknowledgements

No one who contributes to this article does not meet the criteria authorship.

Author details

College of Engineering, National University of Defense Technology, Hefei, China.

References

1. A.U., K., G., A., Z.H., A., Waqas, M., A.K, H.: Spectrum utilization efficiency in the cognitive radio enabled 5g-based iot(article). Journal of Network and Computer Applications 164, 102686 (2020)

2. Christos, T., Elena, L.-A., George, A., Dimitris, V.: Matching theory as enabler of efficient spectrum management in $5 \mathrm{~g}$ networks. Transactions on Emerging Telecommunications Technologies 31, 1-21 (2020)

3. Youping, Z.: Enabling cognitive radios through radio environment maps. PhD thesis, Virginia Polytechnic Institute and State University (2007)

4. Wang, Y., Wang, Y., Zhang, X., Wang, D., Yan, J.: Modeling of pollutant distribution based on mobile sensor networks. Environmental science and pollution research international 27, 11413-11424 (2020)

5. Denkovski, D., Atanasovski, V., Gavrilovska, L., Riihijärvi, J., Mähönen, P.: Reliability of a radio environment map: Case of spatial interpolation techniques. In: 2012 7th International ICST Conference on Cognitive Radio Oriented Wireless Networks and Communications (CROWNCOM), pp. 248-253 (2012)

6. Suchański, M., Kaniewski, P., Romanik, J., Golan, E., Zubel, K.: Radio environment maps for military cognitive networks: density of small-scale sensor network vs. map quality. EURASIP Journal on Wireless Communications and Networking 2020, 2020 (2020)

7. Liu, X., Chen, F., Lu, C.-T.: Robust prediction and outlier detection for spatial datasets. In: 2012 IEEE 12 th International Conference on Data Mining, pp. 469-478 (2012)

8. Yifan, W., Silong, P., Qiong, X., Pengcheng, X.: Nonlinear least squares with local polynomial interpolation for quantitative analysis of ir spectra. Spectrochimica Acta Part A: Molecular and Biomolecular Spectroscopy 206, 147-153 (2019)

9. Wenwu, Z., et al.: Improved minimum curvature iteration algorithm for intrapolation and extrapolation of gravity profile data. Journal of Geodesy and Geodynamics[Chinese] 39, 810-812 (2019)

10. Gajewski, P.: Propagation models in radio environment map design. In: 2018 Baltic URSI Symposium (URSI), pp. 234-237 (2018)

11. Le Nir, V., Scheers, B.: Multiple jammer localization and transmission power estimation for radio environment map. In: 2018 International Conference on Military Communications and Information Systems (ICMCIS), pp. 1-5 (2018) 
12. LI, J., GAO, Z., PEI, Z.: The radio environment map parameter estimation using kriging method based on propagation model. Journal of Computational Information Systems 11, 7607-7616 (2015)

13. Tsukamoto, K., Kitsunezuka, M., Kunihiro, K.: Highly accurate radio environment mapping method based on transmitter localization and spatial interpolation in urban los/nlos scenario. In: 2018 IEEE Topical Conference on Wireless Sensors and Sensor Networks (WiSNet), pp. 5-7 (2018)

14. Hao-fei, Y., et al.: Reconstruction of gravity /magnetic data with the projection-onto-convex-sets methods. Progress in Geophysics[Chinese] 31, 2192-2197 (2016)

15. Xiaoniu, Z., et al.: Simultaneous interpolation, edge padding and denoising method for gravity data based on the projection onto convex sets. Oil Geophysical Prospecting[Chinese] 55, 197-205 (2020)

16. Zhen, Z., Chen, Y., Wen, L., Han, B.: An intelligent cooperative mission planning scheme of uav swarm in uncertain dynamic environment. Aerospace Science and Technology 100, 105826 (2020)

17. BJ, F., D, D.: Clustering by passing messages between data points. Science 315, 972-976 (2007)

18. Lophaven, S.N., Nielsen, H.B., Søndergaard, J.: Dace-a matlab kriging toolbox. PhD thesis, Technical University of Denmark (2002) 


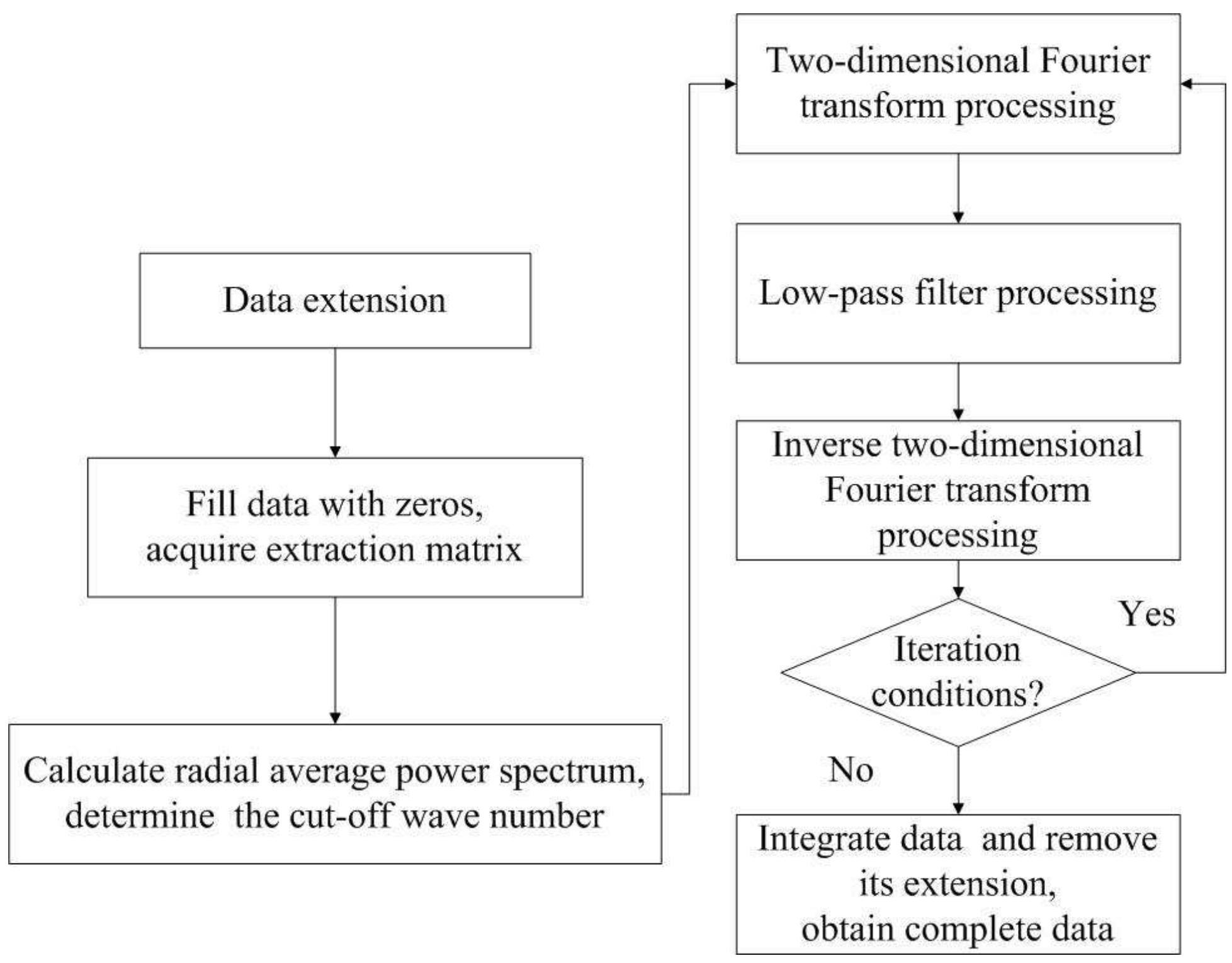

Figure 1

Data processing procedure of domain mapping 


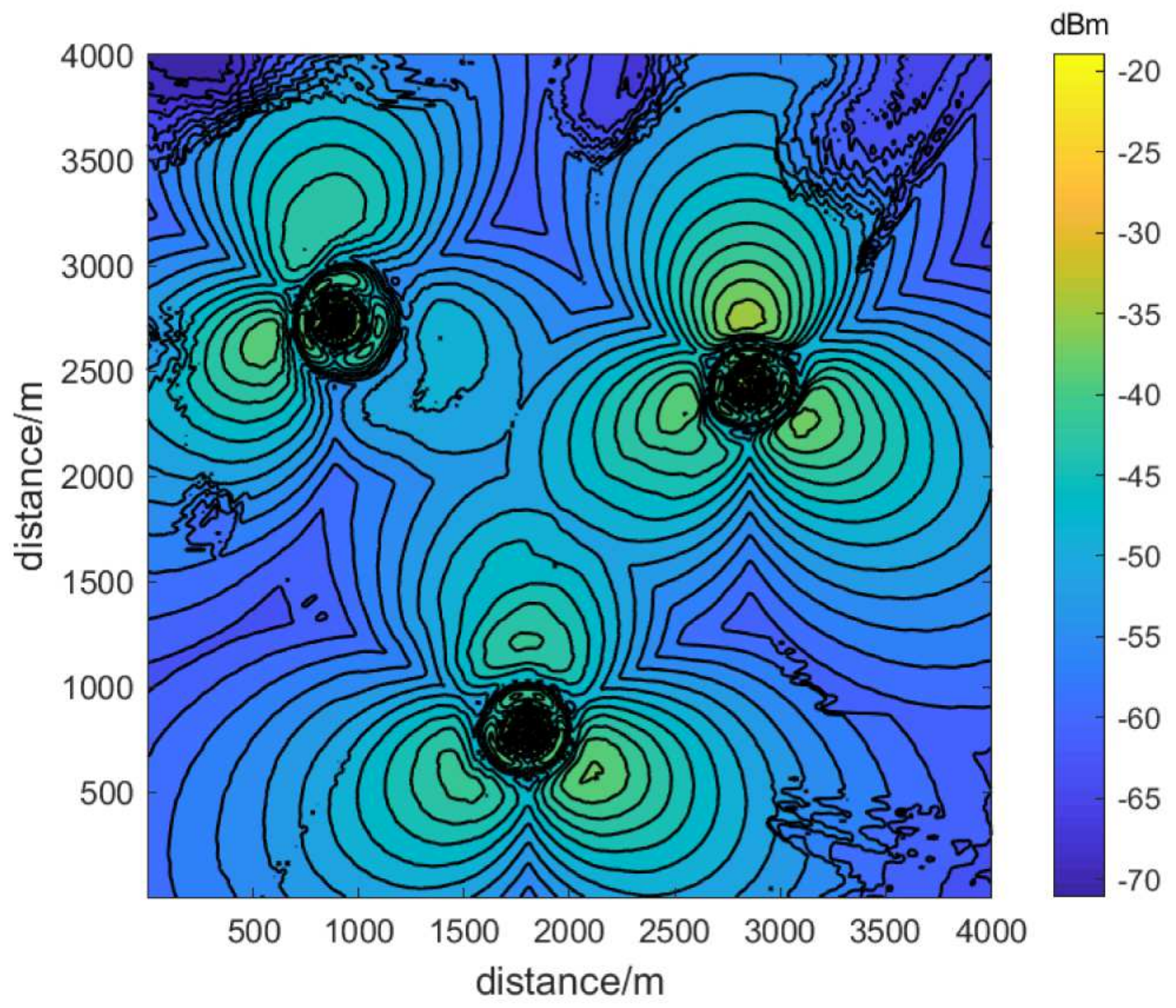

Figure 2

The original electromagnetic map 


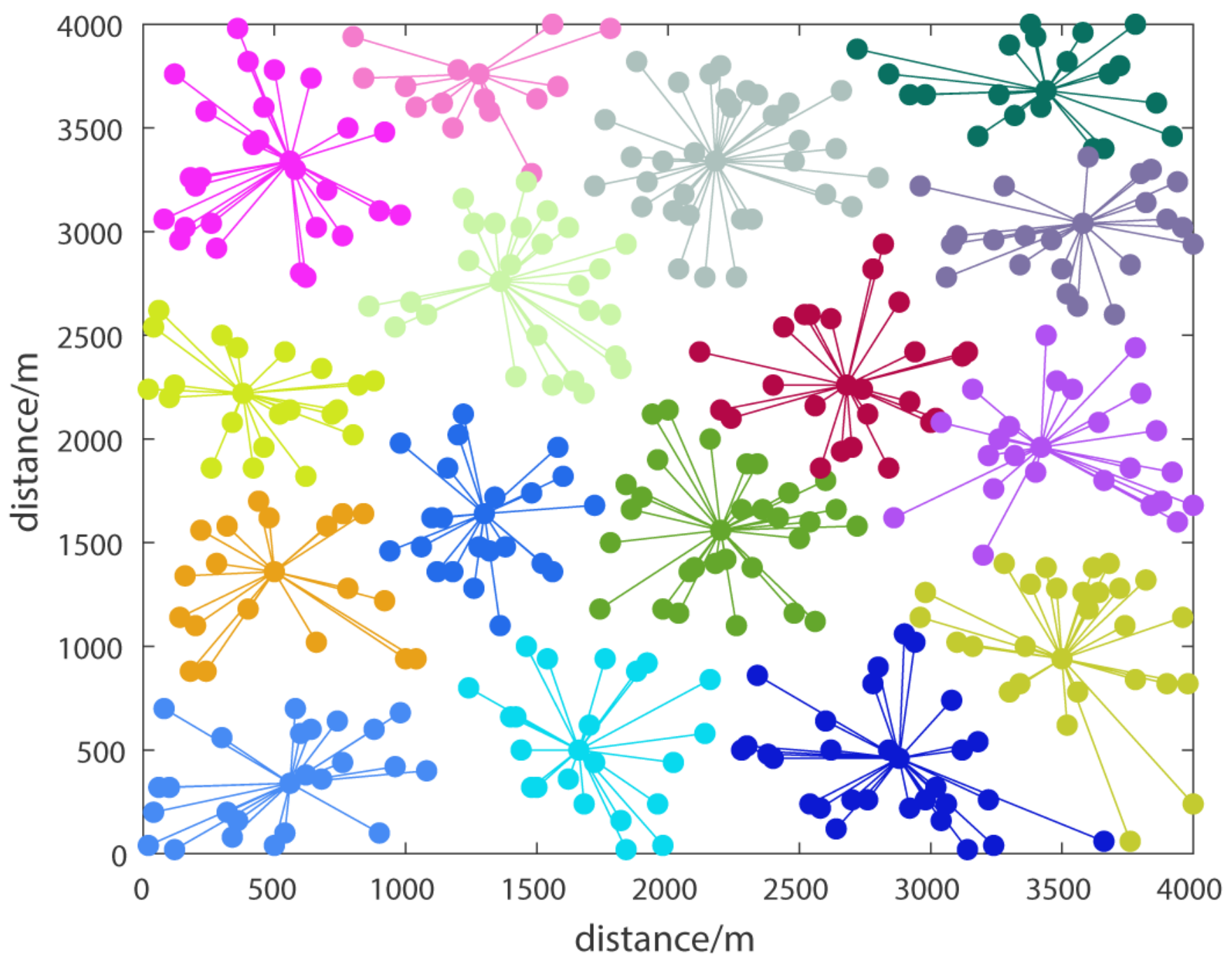

Figure 3

Results of clustering 


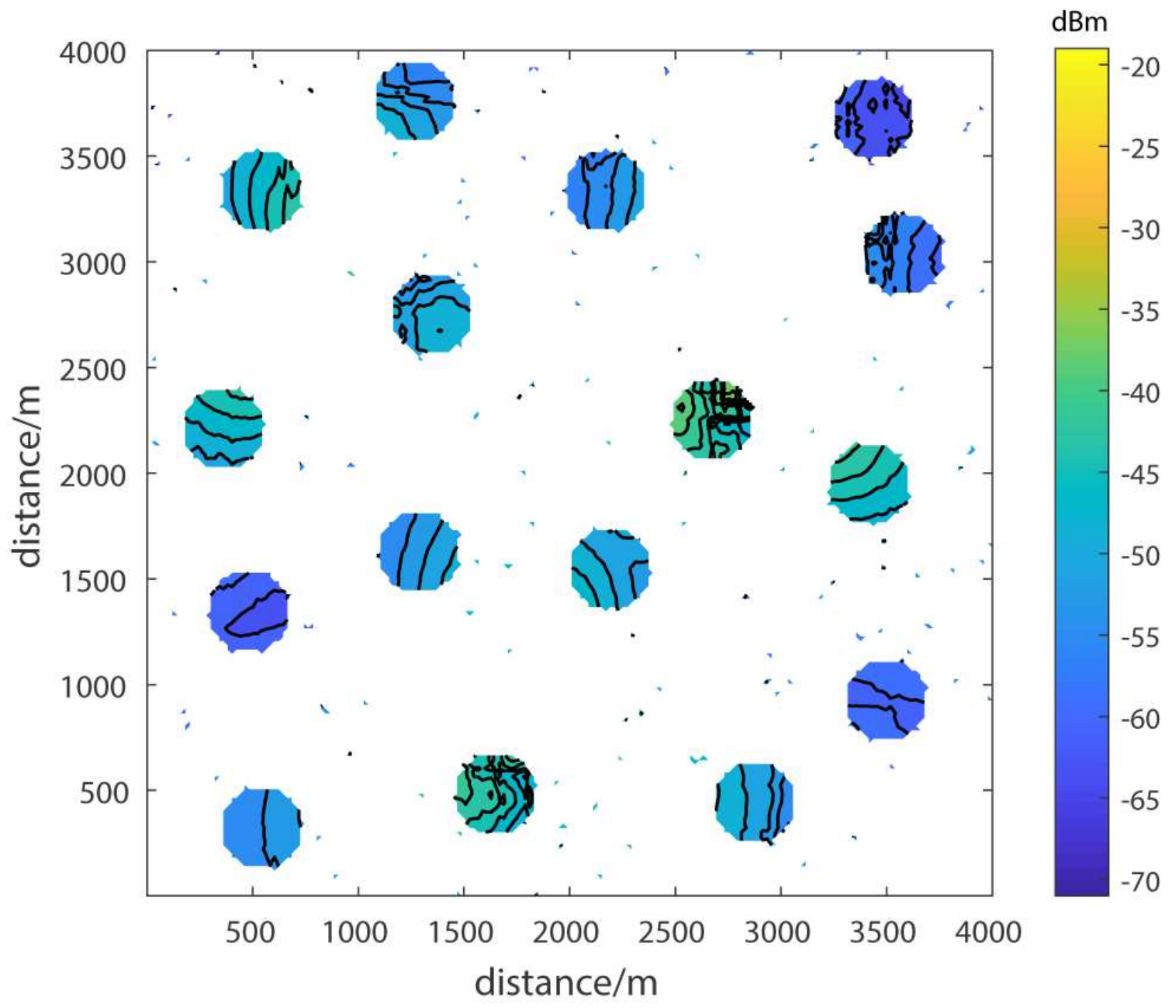

Figure 4

The reconstruction of small-area electromagnetic maps. 


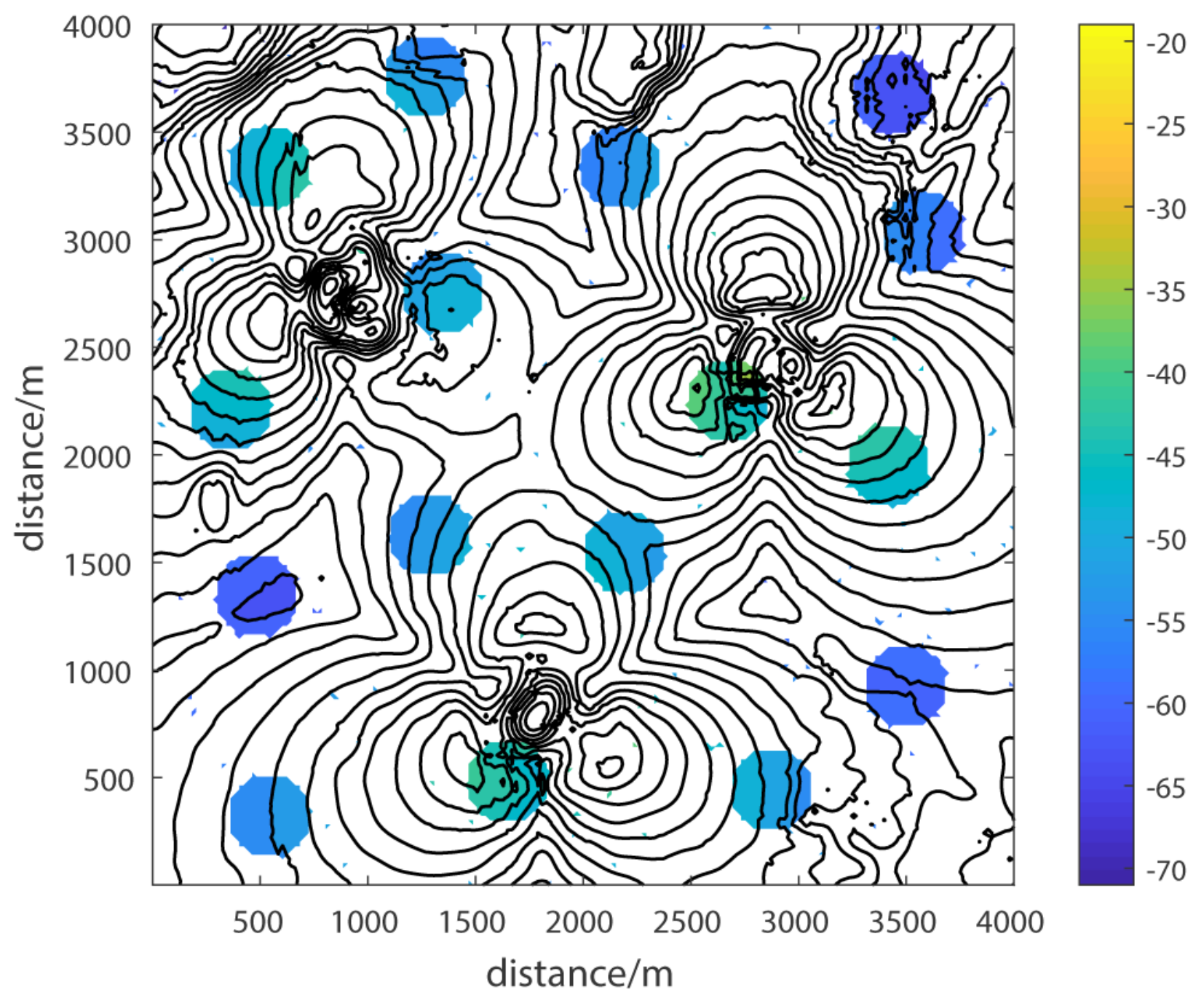

Figure 5

The result of DPA. 


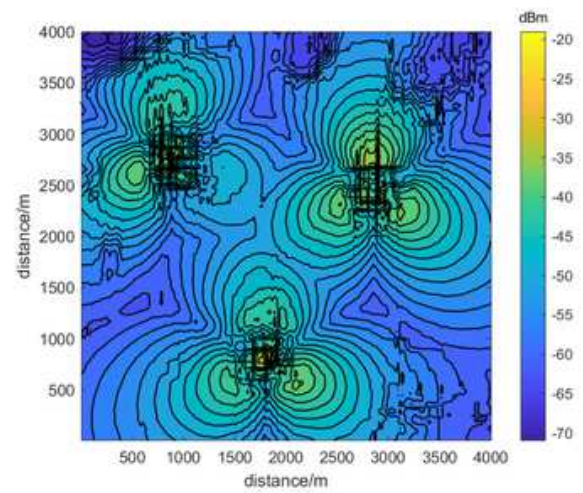

A

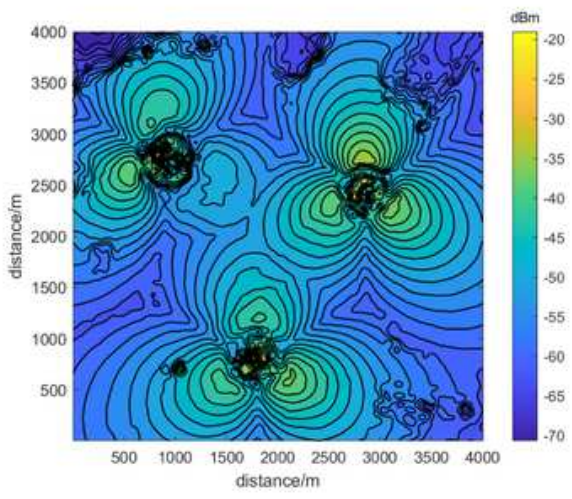

C

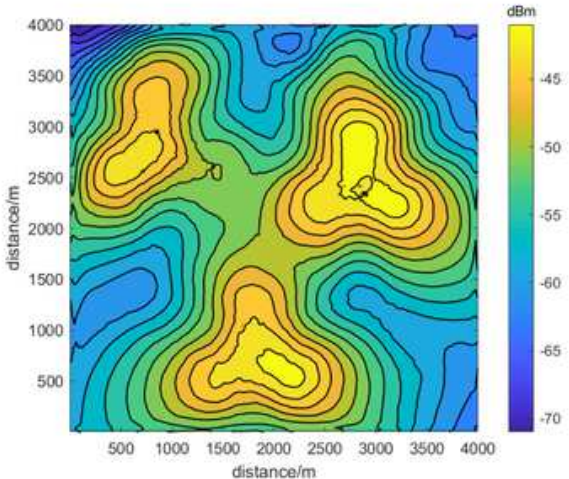

E

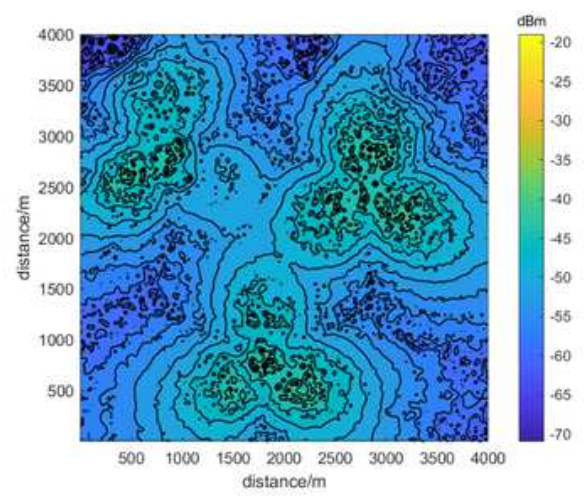

B

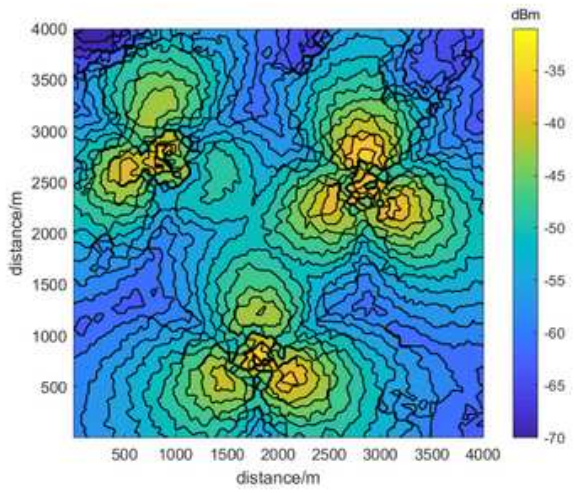

D

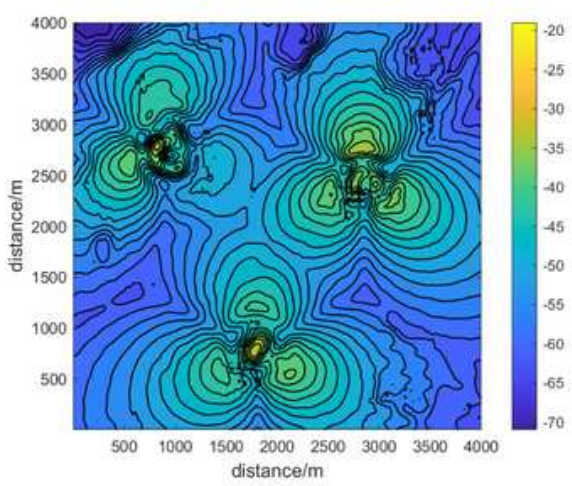

F

Figure 6

Comparative results of reconstruction 


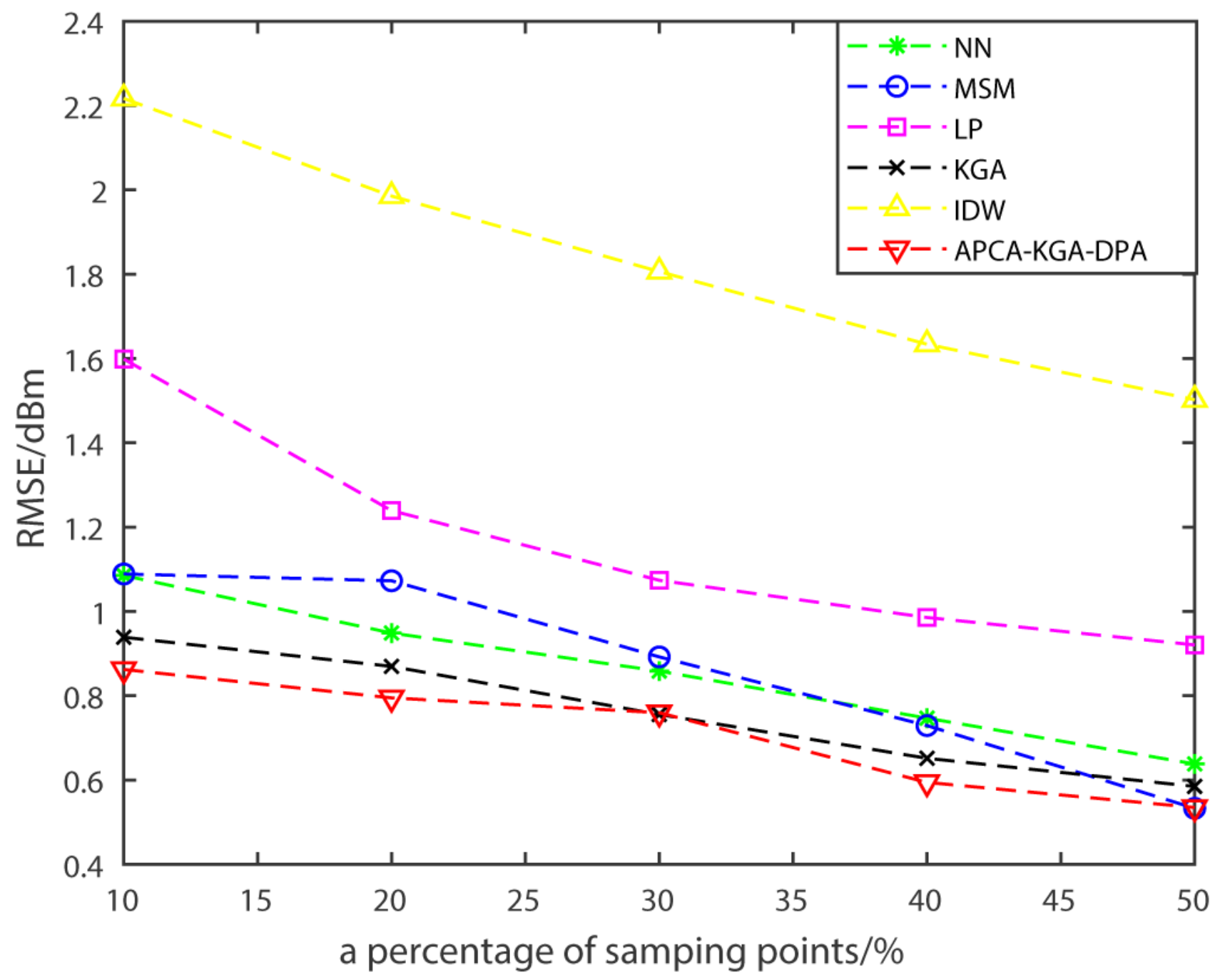

Figure 7

Analyses of robustness 1 


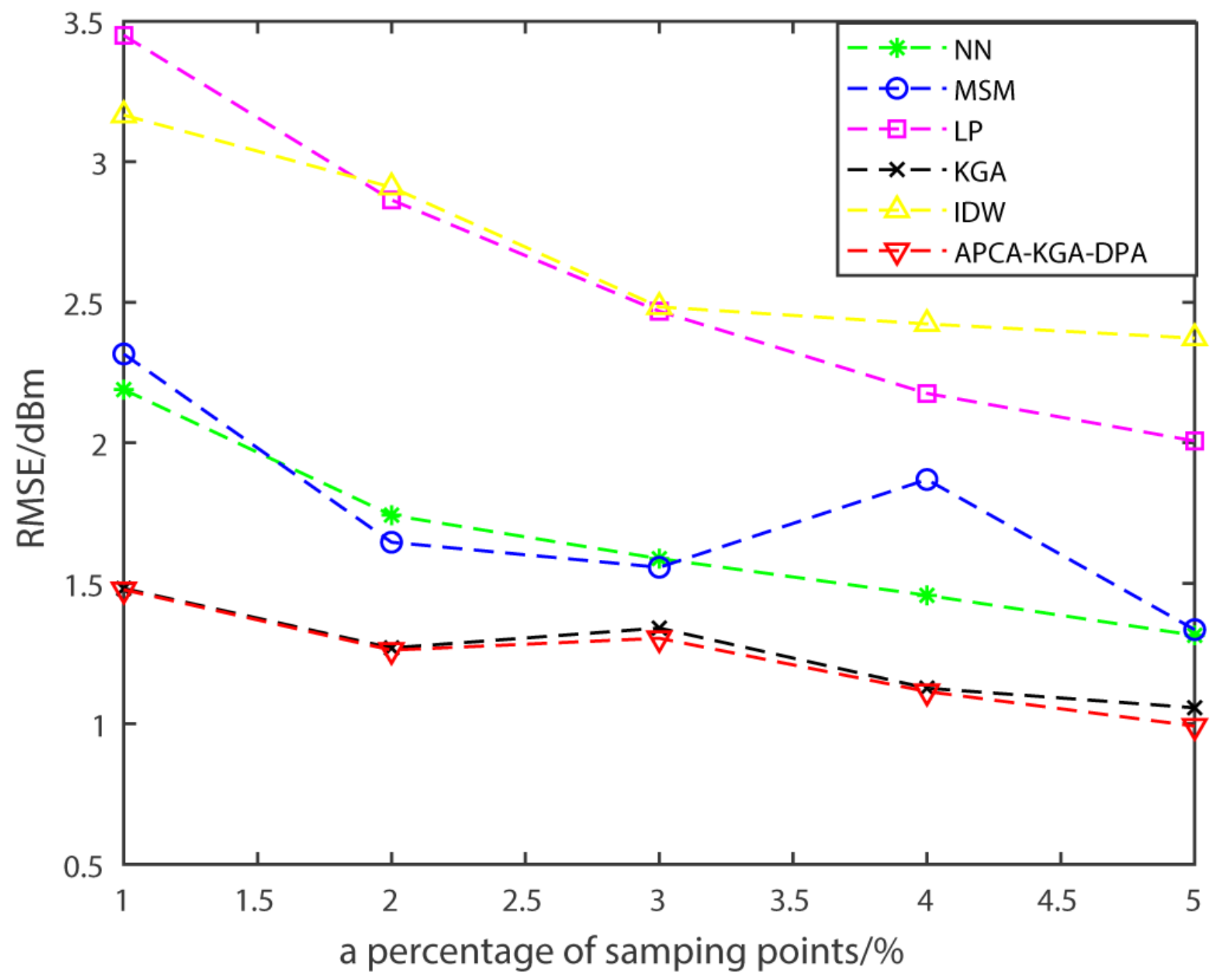

Figure 8

Analyses of robustness 2 